\title{
Supporting Information: Deep Learning of Binary Solution Phase Behavior of Polystyrene
}

Jeffrey G. Ethier ${ }^{1,2}$, Rohan K. Casukhela ${ }^{1,2}$, Joshua J. Latimer ${ }^{1,2}$, Matthew D. Jacobsen ${ }^{1}$, Allen B. Shantz ${ }^{1}$, and Richard A. Vaia*1

${ }^{1}$ Materials and Manufacturing Directorate, Air Force Research Laboratory, Wright-Patterson AFB, OH, 45433

${ }^{2}$ UES, Inc., Dayton, Ohio 45431

*Corresponding Author: richard.vaia@us.af.mil

\section{Contents}

1 Polystyrene Data Set $\quad$ S1

2 Solvent Features $\quad$ S4

2.1 Hansen Solubility Parameters $\quad$ S4

2.2 Solvent Fingerprinting $\quad$ S5

3 Summary of Feature Vector $\quad$ S6

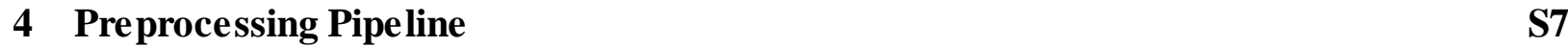

5 Neural Network $\quad$ S8

5.1 Architecture $\quad$ S8

5.2 Train/Test Split Results $\quad$ S11

5.3 Solvent Feature Comparison $\quad$ S13

6 Gaussian Process Regression $\quad$ S20

$\begin{array}{ll}\text { 6.1 Model Details } & \text { S20 }\end{array}$

6.2 GPR Model Performance $\quad$ S21

6.3 Predictions for PS/Cyclohexane $\quad$ S26

7 Experimental Details : PS in Cyclopentane S29

8 Additional Computational Details $\quad$ S30

9 References $\quad$ S31 


\section{Polystyrene Data Set}

Solubility curves for polystyrene in 19 different solvents were collected from the CRC Handbook of Liquid-Liquid Equilibrium Data of Polymer Solutions. ${ }^{1}$ A PDF version of the book was parsed into a custom JSON-structured file using Python's pyPdf module and custom-built Python functions. Only binary coexistence data was processed and thus, each entry in the JSON database is a single entry from the binary section of the CRC Handbook. The JSON structure was then converted to a CSV formatted data set where each row contains information for one cloud point temperature. To make reported polymer concentration values consistent across all references cited in the CRC Handbook, we chose to convert all polymer weight fractions to volume fraction by,

$$
\phi=\frac{w_{p} / \rho_{p}}{w_{p} / \rho_{p}+w_{s} / \rho_{s}}
$$

where $\mathrm{w}_{\mathrm{i}}$ and $\rho_{\mathrm{i}}$ is the weight fraction and density, respectively, with ' $\mathrm{i}$ ' being either polymer ' $\mathrm{p}$ ' or solvent ' $\mathrm{s}$ '. The density of polystyrene used was $1.05 \mathrm{~g} / \mathrm{cm}^{3}$. Various solvent densities and their Hansen solubility parameters (HSPs) are reported in Table S1. Additionally, a pressure of $0.1 \mathrm{MPa}$ (atmospheric) was recorded unless otherwise specified and units of bar were converted to MPa.

Within the CRC Handbook, binary solubility data was either reported as "cloud point" or as "coexistence data", where the former reports the total polymer concentration for the cloud point and the latter reports the concentrations in the gel and solution phase. Both types of data are included in the polystyrene data set, but entries with only one recorded cloud point were excluded. Additionally, data is explicitly reported as "UCS" (upper critical solubility) or "LCS" (lower critical solubility) behavior. Curves that are not explicitly labelled as such are added manually depending on the ability to determine the one-phase temperature region. This means that some data were excluded, including 5 references from the CRC Handbook in which the one-phase region was unclear. Furthermore, cloud points near the hypercritical point (at which the UCS and LCS branches merge) are excluded as the one-phase region is only accessible with a change in pressure for poor solvents and would require an expansion of the one-phase direction feature (see Table $\mathrm{S} 2$ ). As the models are expanded to more complex systems, this descriptor should be expanded to a unit vector in phase space that points to the one phase region (in $T-\phi-P$ phase space), such as a one-hot encoded variable. The additional complexity and lack of data does not justify such inclusions at this point. Lastly, we calculate the polydispersity $\left(\mathrm{PDI}=M_{\mathrm{w}} / M_{\mathrm{n}}\right)$ and discard any 
entries with PDI greater than 6. The total number of cloud points and polymer molecular weights used for model training are summarized for each solvent and UCS/LCS branch in Figure S1. The CSV-formatted PS data set along with the trained models can be accessed on Google Cloud Platform with user permission. Users interested in the accessing the data set and/or model predictions can contact the corresponding author.

Table S1. Hildebrand parameter, Hansen solubility parameters, and density $\left(\mathrm{g} / \mathrm{cm}^{3}\right)$ listed for each solvent used in the polystyrene data set. Density values are taken from PubChem ${ }^{2}$ at $20{ }^{\circ} \mathrm{C}$ unless otherwise noted. For reference, values for polystyrene are listed at the bottom.

\begin{tabular}{cccccc}
\hline Solvent & $\boldsymbol{\delta}$ & $\boldsymbol{\delta}_{\mathbf{D}}$ & $\boldsymbol{\delta}_{\mathbf{P}}$ & $\boldsymbol{\delta}_{\mathbf{H}}$ & $\rho$ \\
\hline 1-dodecanol* & 18.93 & 16 & 4 & 9.3 & 0.831 \\
2-propanone & 19.67 & 16.18 & 9.07 & 6.55 & 0.791 \\
2,2,4-trimethylpentane & 14.10 & 14.1 & 0 & 0 & 0.692 \\
butyl acetate & 17.41 & 15.8 & 3.7 & 6.3 & 0.875 \\
cyclohexane & 16.80 & 16.8 & 0 & 0.2 & 0.779 \\
cyclohexanol & 22.40 & 17.4 & 4.1 & 13.5 & 0.947 \\
cyclopentane & 16.50 & 16.4 & 0 & 1.8 & 0.745 \\
diisodecyl phthalate & 17.91 & 16.6 & 6.2 & 2.6 & 0.967 \\
ethyl acetate & 18.15 & 15.8 & 5.3 & 7.2 & 0.902 \\
hexyl acetate & 14.74 & 14.7 & 1.1 & 0 & 0.876 \\
methyl acetate & 18.70 & 15.5 & 7.2 & 7.6 & 0.927 \\
methylcyclohexane & 16.03 & 16 & 0 & 1 & 0.766 \\
n-hexane & 14.90 & 14.9 & 0 & 0 & 0.659 \\
nitroethane & 22.73 & 16 & 15.5 & 4.5 & 1.05 \\
n-pentane & 15.60 & 15.6 & 0 & 0 & 0.63 \\
pentyl acetate & 17.26 & 15.8 & 3.3 & 6.1 & 0.876 \\
propionitrile & 21.65 & 15.3 & 14.3 & 5.5 & 0.8 \\
tert-butyl acetate & 16.57 & 15 & 3.7 & 6 & 0.8665 \\
trans-decahydronaphthalene & 17.60 & 17.6 & 0 & 0 & 0.87 \\
\hline Polymer & $\boldsymbol{\delta}$ & $\boldsymbol{\delta}_{\mathbf{D}}$ & $\boldsymbol{\delta}_{\mathbf{P}}$ & $\boldsymbol{\delta}_{\mathbf{H}}$ & $\rho$ \\
\hline polystyrene & 19.26 & 18.5 & 4.5 & 2.9 & 1.05 \\
\hline
\end{tabular}




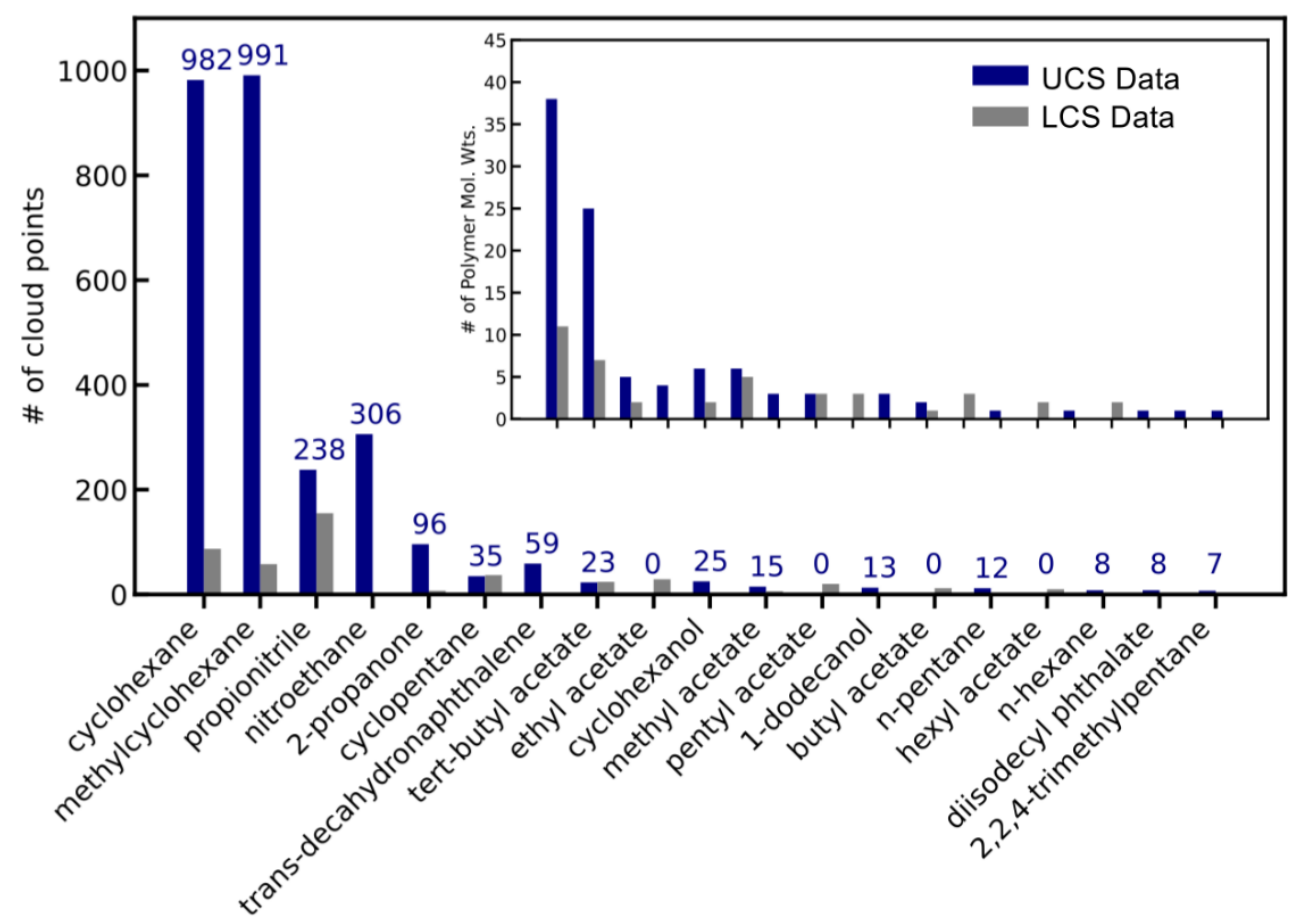

Figure S1. Number of polystyrene cloud points in various solvents. The total UCS data is listed above each solvent bar. Inset: Number of molecular weight values for UCS (blue) and LCS (gray) curves for each solvent. 


\section{Solvent Features}

As discussed in the manuscript, a solvent feature is required to train the model to learn the varying cloud point ranges and coexistence behavior. For instance, for polystyrene UCS behavior, phase separation in some solvents such as 2,2,4-trimethylpentane and n-hexane occurs at temperatures below $0{ }^{\circ} \mathrm{C}$, whereas in solvents such as 1 -dodecanol, cloud points appear above $100{ }^{\circ} \mathrm{C}$ (see Figure S5). For the model to capture these differences, a numeric representation must be used to describe the solvent quality. Below, we discuss the use of empirical surrogate parameters (e.g., physical properties such as Hansen solubility parameters) to topological "fingerprints" based on the molecule structure.

\subsection{Hansen Solubility Paramete rs}

To ensure our models encapsulate the effects of various solvents, polymer-solvent affinity must be encoded properly. Specifically, for our cloud point curves a solvent feature is required to model the different affinities between polystyrene and the various solvents. Previously, solubility of a polymer in solvent is determined via their "solubility parameters". This first began with the Hildebrand solubility parameter $\delta$, a single parameter based on the cohesive energy densities of the pure components and describes the total van der Waals forces of a molecule. ${ }^{3,4}$ This was later expanded to the more widely-accepted, three-component Hansen solubility parameters (HSPs) developed by Charles Hansen to include effects of polarity and hydrogen bonding. ${ }^{5}$ The three HSP components $\left(\delta_{D}, \delta_{P}, \delta_{H}\right)$ are additive and their parts sum to the Hildebrand parameter. However, the Hildebrand parameter is more widely available and for a larger number of polymers, despite the lack of accuracy in predicting solvents/nonsolvents for polar molecules. ${ }^{6}$ Typically, solvents and nonsolvents for a select polymer are chosen based on their relative energy distance (RED), defined as the absolute distance between their HSP values (in 3-dimensional space). HSP values have worked well in describing the solubility of polymers in select solvents. However, solubility parameters are unable to generate the full temperature and concentration-dependent coexistence behavior. Hence, we aim to use them solely as the solvent feature to distinguish the nonlinear coexistence behavior of various solvents. 


\subsection{Solvent Finge rprinting}

A commonly implemented molecule descriptor for machine learning (ML) in materials science is the topological "fingerprint", which encodes the molecular structure into a high-dimensional binary vector. Molecular fingerprints have had success in ML models for polymers. ${ }^{7}$ Recently, molecular fingerprints have been used with neural networks to predict a polymer's solvent/nonsolvent. ${ }^{8}$ The authors showed ML models perform better in predicting solvent/nonsolvent, compared to standard methods such as calculating the RED from Hansen solubility parameters. This improvement is due to the fact that HSP values for polymers are mostly unknown and/or vary based on experimental methods, which suggests molecular fingerprint descriptors work well in describing the affinity between solvent and polymer for which HSP values are unknown. In the main manuscript, we use a 3-dimensional vector containing HSP values as the solvent descriptor, and below we show a DNN model that uses a 2048- and 64-dimensional vector containing the solvent's topological "fingerprint".

The fingerprint is generated using a Daylight-like algorithm implemented by the RDKit open-source cheminformatics package. ${ }^{9}$ A solvent molecule is first read into RDKit via its InChI string. The algorithm then splits the molecule structure into fragments of units containing 1 to 7 bonds and hashes each fragment to encode all possible connectivity pathways. The result is a $\mathrm{N}$ bit binary vector containing $0 \mathrm{~s}$ and $1 \mathrm{~s}$. We treat each bit as a solvent feature, resulting in over $N$ features (combined with the polymer and state features) as input to the neural network. The default fingerprint size of $N=2048$ bits is compared to $N=64$ bits (the minimum that RDKit allows) in Section 5.3 below. 


\section{Summary of Feature Vector}

The JSON-structured database was converted to a Pandas DataFrame before preprocessing for training the ML models. Each column in the DataFrame consists of a single feature from the set of polymer, solvent, and state features, and each row corresponds to a single training instance. The input feature vector consists of the one-phase direction ("0" or "1"), solvent $\operatorname{HSPs}\left(\delta_{D}, \delta_{P}, \delta_{H}\right)$ or topological fingerprint, polymer molecular weight $\left(M_{\mathrm{w}}\right)$, polymer volume fraction $(\phi)$, polymer polydispersity index (PDI), pressure (units of $\mathrm{MPa}$ ), and cloud point temperature (units of ${ }^{\circ} \mathrm{C}$ ). The list of Hansen solubility parameters $\left(\delta_{D}, \delta_{P}, \delta_{H}\right)$ can be found in Table S1. A summary of the feature vector input to our models is shown in Table S2. If other linear polymer data is included other than polystyrene, an addition polymer feature (either HSP or fingerprint) is required.

Table S2. Feature vector components consisting of polymer, solvent, and state features.

\begin{tabular}{|c|c|c|c|}
\hline Polymer & Solvent & State & Target \\
\hline $\begin{array}{c}\text { Weight-average } \\
\text { molecular weight, } M_{\mathrm{w}}\end{array}$ & $\begin{array}{c}\text { Hansen Solubility Parameters } \\
\text { OR } \\
\text { Solvent Fingerprint }\end{array}$ & $\begin{array}{l}\text { One-phase direction } \\
\text { ( } 0 \text { or } 1)\end{array}$ & $\begin{array}{l}\text { Cloud point } \\
\text { temperature }\end{array}$ \\
\hline Volume fraction $(\phi)$ & & Pressure & \\
\hline Polydispersity $\left(M_{\mathrm{w}} / M_{\mathrm{n}}\right)$ & & & \\
\hline
\end{tabular}




\section{Preprocessing Pipeline}

The polystyrene data set above was split into a training set and test set. As mentioned in the main manuscript, a stratified sampling method was employed to remove bias from the uneven distribution of UCS and LCS cloud points. Specifically, we set aside $10 \%$ of each solvent's UCS and LCS data for the test set. The remaining $90 \%$ was subjected to a preprocessing pipeline before training the deep neural network (DNN) and Gaussian process regression (GPR) models. First, polymer molecular weight and volume fraction were converted to their log values. This reduced the molecular weight values (which can span many orders of magnitude) to a reasonable scale and removed the large slope at low concentration values. Secondly, all numerical columns were then standardized to have a mean of 0 and standard deviation of 1 (including the HSP values). This is done to improve the performance of neural networks during back propagation. ${ }^{10}$ Statistics for each column feature (mean and standard deviation) are then saved for transforming the test set before calculating performance metrics. 


\section{Neural Network}

\subsection{Architecture}

We test several different neural network models to determine an optimal architecture (i.e., the number of hidden layers and neurons per layer). First, the 80:10:10 stratified split is used for the training, validation, and test data sets. The training and validation data sets are used to update the DNN weights during training. The DNN is constructed with 1 to 4 hidden layers, with each layer containing either 56, 64, 128, or 256 neurons per layer. The input to the DNN is the above feature vector containing either HSPs as the solvent feature or the 2048-bit solvent fingerprint, where we compare the performance of both. The connection weights of each layer are initialized using "He" initialization and the output of each neuron is the 'ReLU' activation function. The output of the DNN is a single neuron with a linear activation function that predicts cloud point temperature (in degrees Celsius). During training, all of the connection weights are updated via the "Adam" optimizer for a maximum of 2000 epochs. To reduce overfitting, we implement early-stopping during training, which stops the training when the validation loss (MSE) does not decrease after 100 epochs from its minimum value. The batch size for training was set to 128 and the optimizer learning rate was set to 0.001 .

In Figures S2 and S3 we explore the root mean square error (RMSE) of the trained DNN model using predictions from the training (train and validation) data set and test set. As the number of hidden layers increases, the RMSE values decrease as expected. Comparing the various 3 hidden layer models (with different neurons in the first and last layer, Figure S2), the number of neurons in the first or last layer do not affect the RMSE values for the UCS predictions, but the RMSE values decrease significantly for the LCS predictions (Figure S2b and S3b). As a result, the optimal architecture is found to be 3 hidden layers with 256, 128, and 56 neurons per layer, sequentially, for both solvent features. Training curves for these models are shown in Figure S4. The loss value (MSE) decreases to less than 10 after about 200 epochs when using HSP values as the solvent features, whereas it takes about 400 epochs to reach the same RMSE using solvent fingerprints. Both training curves plateau at about the same RMSE value, with the validation RMSE value stabilizing at a slightly larger value than the training RMSE. 
(a)

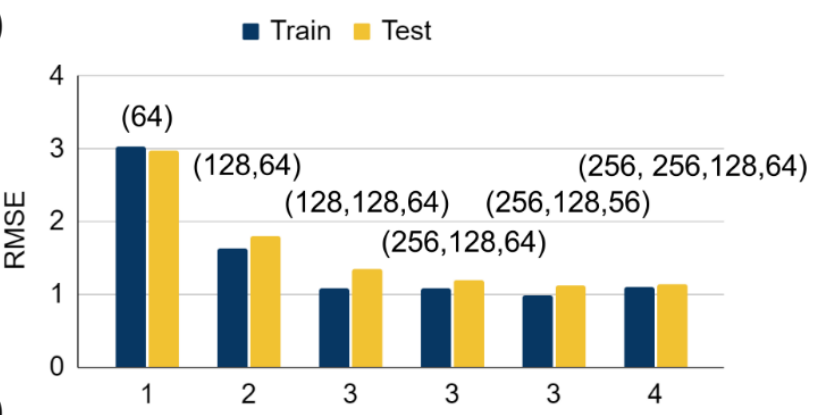

(b)

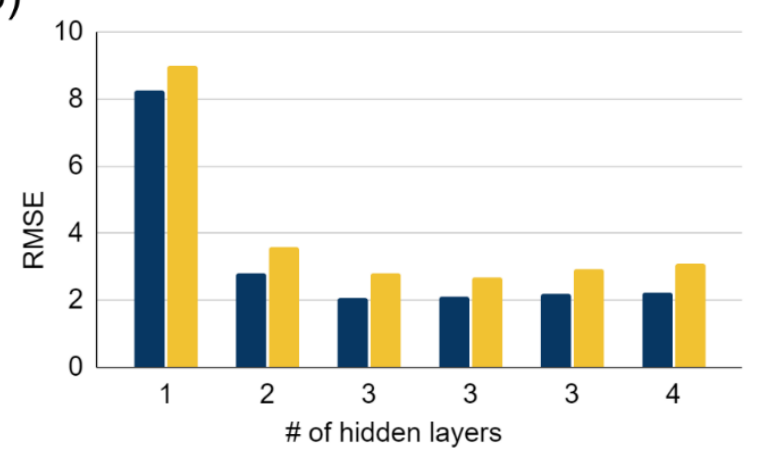

Figure S2. RMSE measured from temperature predictions on the training set (blue) and test set (yellow) for (a) UCS and (b) LCS data and various number of hidden layers. The DNN model was trained using HSPs as the solvent feature with various number of neurons per hidden layer labeled in (a).

(a)

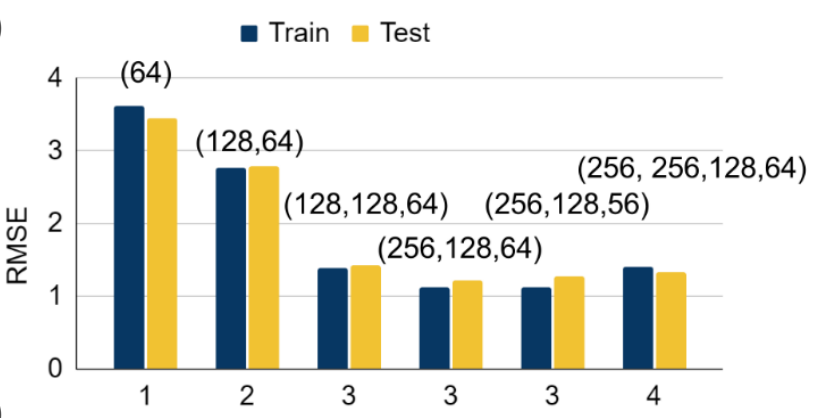

(b)

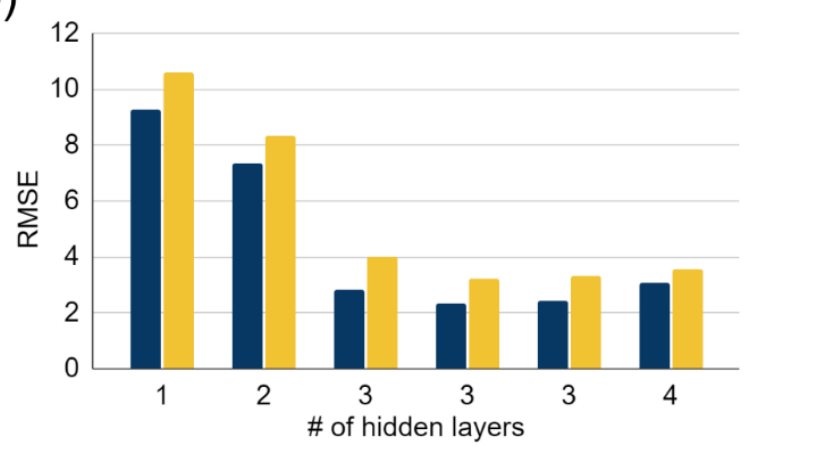

Figure S3. RMSE measured from temperature predictions on the training set (blue) and test set (yellow) for (a) UCS and (b) LCS data and various number of hidden layers. The DNN model was trained using solvent fingerprints $(N=2048$ bits $)$ as the solvent feature with various number of neurons per hidden layer labeled in (a). 
By examining the training curves with the resulting test RMSE and mean absolute error (MAE) values (see Figure 1 in manuscript and Figure S8 below), there appears to be no indication of overfitting. Further analysis of the average train/test split results (Section 5.2) and crossvalidation results (Section 5.3) also suggest the model is not overfitting, which would be apparent if the test MAE values were significantly larger than the training MAE values. However, we expect that for some solvents, generalization to other polymer $M_{\mathrm{w}}$ or extrapolation to low concentrations will be poor due to lack of training data, which is typical of most supervised ML models. Another indication of overfitting is the model fitting noise in the data. For instance, we plot two additional solvent predictions in Figure S5 below. We find that even though there is noise in the data, the predictions do not fit the noise exactly which would be a sign of overfitting.

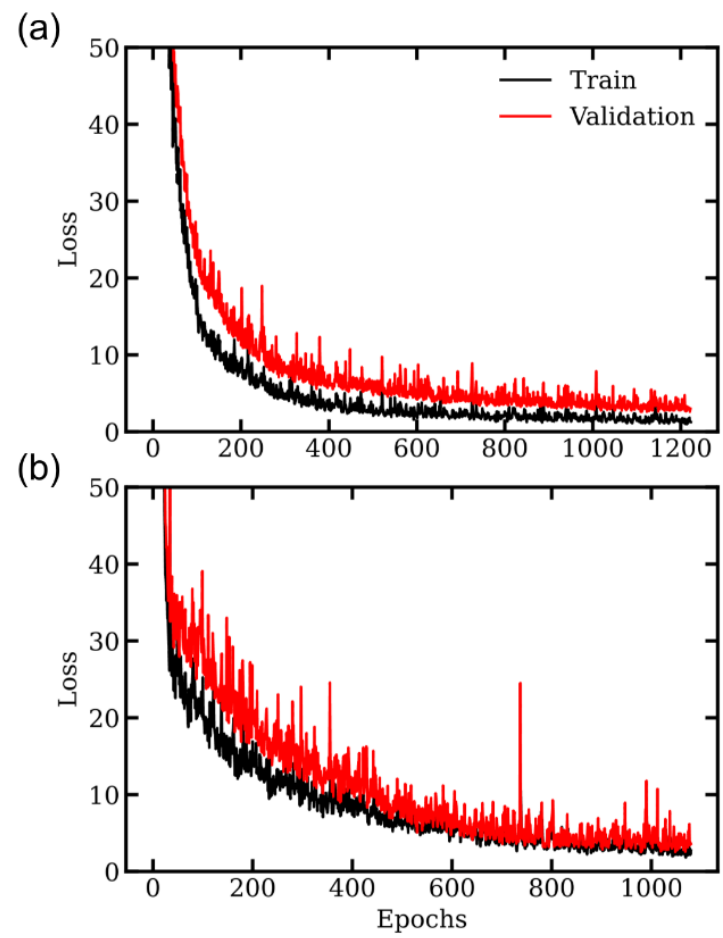

Figure S4. Loss (MSE) curves during DNN model training using (a) HSPs and (b) 2048-bit fingerprints as the solvent feature. The DNN model consists of 3 hidden layers with $(256,128,56)$ neurons in each layer. 
(a)

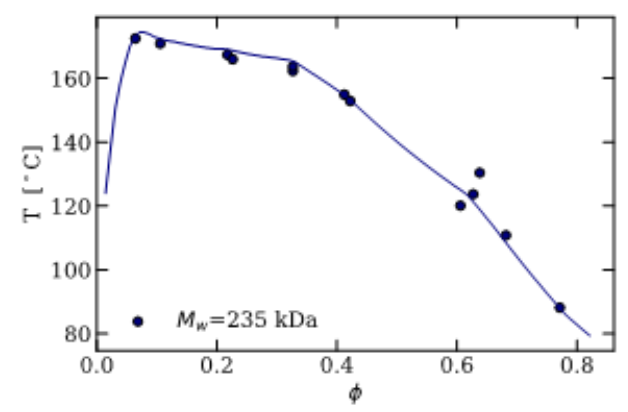

(b)

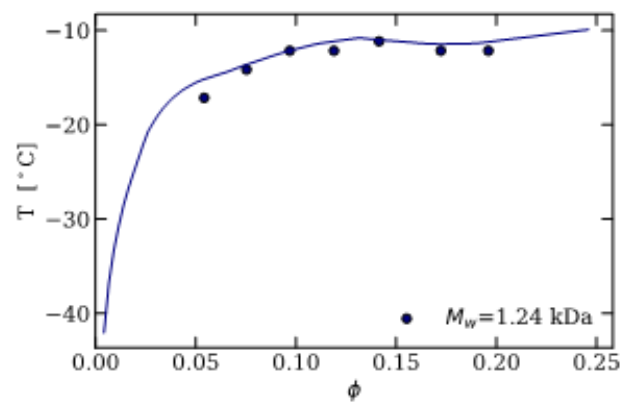

Figure S5. Polystyrene phase behavior in (a) 1-dodec anol and (b) 2,2,4-trimethylpentane. DNN predic tions (lines) are compared to experimental observations (markers). HSPs are used for the solvent feature in the $(256,128,56)$ DNN model.

\subsection{Train/Test Split Results}

In Figure S6, we show the HSP-trained DNN model performance as a function of the train/test split size, ranging from a 60:10:30 split to an 80:10:10 split for the training, validation, and test set, respectively. The predicted RMSE values on the training data set is not affected by the training set size. However, it is clear that as the training set size increases, the test set RMSE values decrease and therefore the model can generalize better on the remaining data (up to $~ 320$ cloud points). Predictions for the LCS data are slightly worse regardless of the split due to the smaller number of training data. Therefore, we choose to train our models using the 80:10:10 stratified split.

Table S3 summarizes the overall metrics comparing the two train/test split methods (stratified $v s$. random) averaged over 50 different splits. We observe a slight decrease in the test RMSE for both UCS and LCS predictions with the stratified split, due to the training data being more representative of the entire data set. In Figure S7, we show the test MAE values for each solvent. The HSP solvent feature was used to train the DNN. As expected, solvents with little data have the largest MAEs and standard deviations. Solvents such as cyclohexane, methylcyclohexane, and nitroethane (with the most amount of cloud points) have the smallest error. Interestingly, we see that some solvents with less data such as n-pentane and 2,2,4trimethylpentane have an MAE less than 2. However, the number of test data points is less than 5 for these solvents due to the small number of training data and thus, may not generalize well to other molecular weights. Nevertheless, the MAE values per solvent suggest that this model can 
predict the test cloud points for each solvent within $4{ }^{\circ} \mathrm{C}$ on average. In most cases, the stratified sampling method reduces the MAE compared to random sampling. Hence, we use the stratified sampling method to ensure the test data includes cloud points from all solvents.
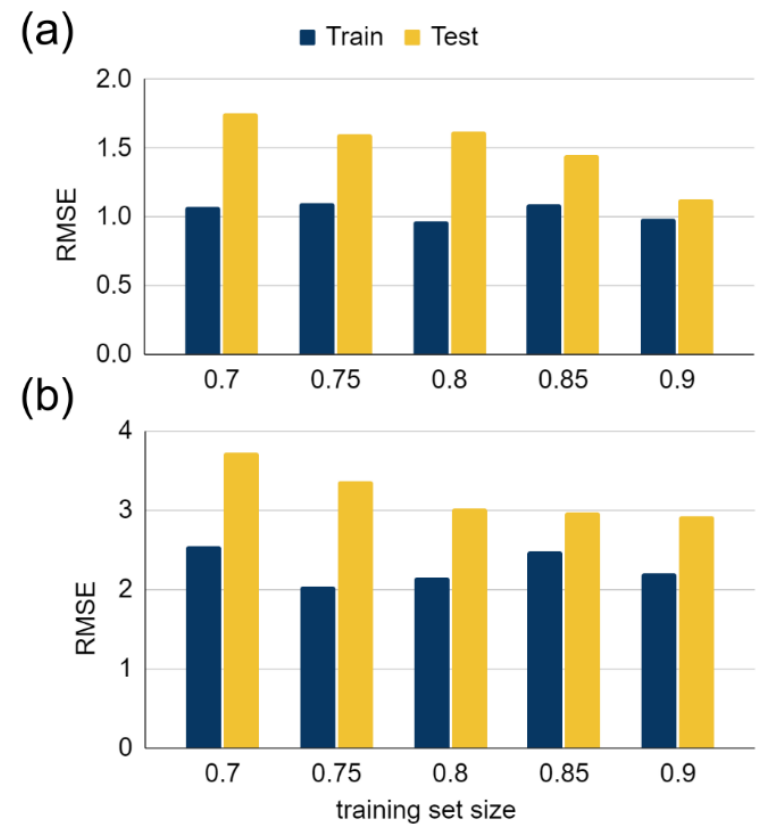

Figure S6. RMSE values for (a) UCS and (b) LCS cloud point predictions on the training set (blue) and test set (yellow) using various training set sizes (fraction of PS data set) split for the train/validation/test data set. HSP values were used as the solvent feature and the DNN model consisted of 256, 128, and 56 neurons per hidden layer.

Table S3. Train and test RMSE/MAE reported for all UCS (top) and LCS (bottom) solubility curves. Stratified split takes $10 \%$ of each solvent's UCS and LCS data to use as the test set. Random split is $10 \%$ of the entire PS data set.

\section{UCS}

\begin{tabular}{|c|c|c|c|c|}
\hline Train/Test Split & Train RMSE & Test RMSE & Train MAE & Test MAE \\
\hline Stratified & $1.08 \pm 0.15$ & $1.49 \pm 0.29$ & $0.61 \pm 0.09$ & $0.79 \pm 0.10$ \\
\hline Random & $1.15 \pm 0.23$ & $1.61 \pm 0.34$ & $0.67 \pm 0.14$ & $0.87 \pm 0.17$ \\
\hline
\end{tabular}

\section{LCS}

\begin{tabular}{|c|c|c|c|c|}
\hline Train/Test Split & Train RMSE & Test RMSE & Train MAE & Test MAE \\
\hline Stratified & $2.26 \pm 0.40$ & $3.66 \pm 0.99$ & $1.48 \pm 0.28$ & $2.38 \pm 0.40$ \\
\hline Random & $2.48 \pm 0.72$ & $3.73 \pm 1.35$ & $1.65 \pm 0.52$ & $2.50 \pm 0.67$ \\
\hline
\end{tabular}




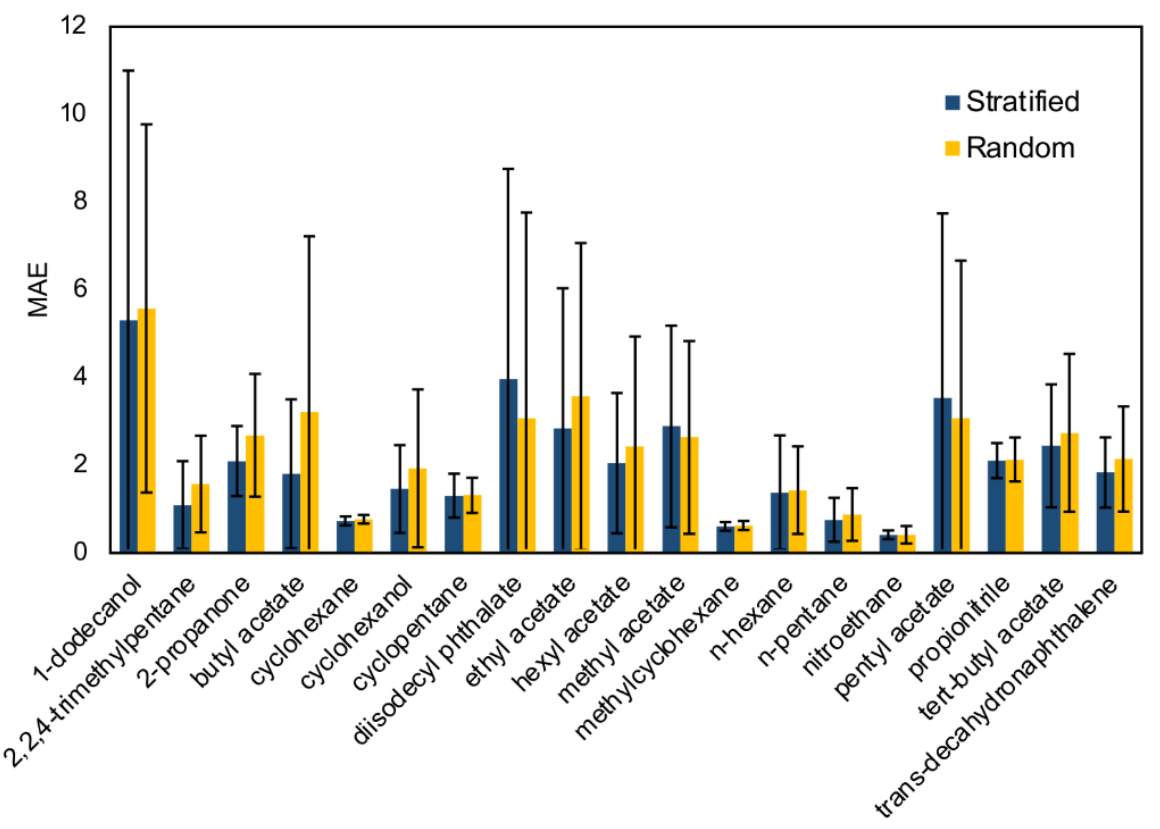

Figure S7. MAE for each solvent's test data using the DNN model and comparing a stratified split (blue) to the random split (yellow) of the entire data set. HSPs are used as the solvent feature during training.

\subsection{Solvent Feature Comparison}

Figure S8 summarizes the neural network performance using the 2048-bit fingerprint as the solvent feature (see manuscript for results using the HSP solvent features). The train/test RMSE and MAE are reported in the figure. The performance of the model shows that either solvent feature is viable and using solvent fingerprints may be used in cases where the HSP values are unknown. This is especially crucial when extending this model to various polymers in which we do not know their HSP values. However, training time is significantly increased due to the size of the feature vector (2053 features). Compared to the HSP-trained DNN, the training time is $2.5 \mathrm{x}$ longer ( $\sim 9$ epochs per second vs. $\sim 22$ epochs per second for the HSP model, see section 8 for hardware details). To reduce the training time, we also compare the 2048-bit solvent feature to a 64-bit solvent feature (Figure S9). The parity plots in Figure S9 show that the 64-bit fingerprints work similarly well. Additionally, training time is significantly reduced ( 20 epochs per second) and is only about a $10 \%$ increase compared to the HSP model. 
(a)

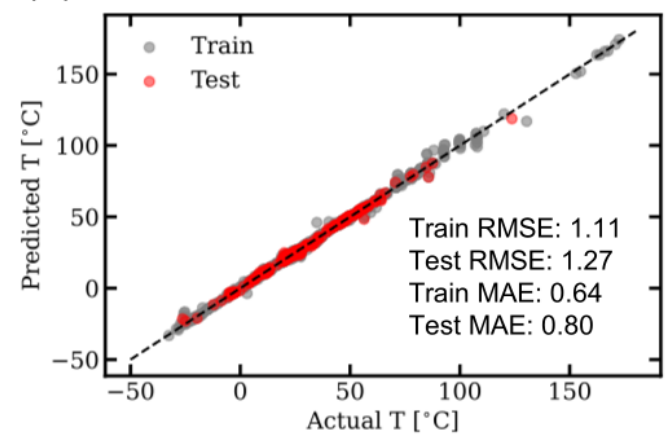

(b)

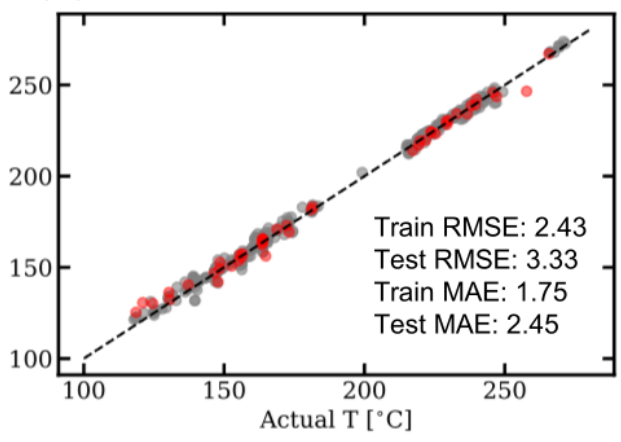

Figure S8. Parity plots for the DNN predictions on the training set (gray) and test set (red) for all (a) UCS and (b) LCS cloud point temperatures using 2048-bit fingerprints as the solvent feature. RMSE and MAE values are reported in each plot. DNN architecture consists of three hidden layers with 256, 128, and 56 neurons per layer in sequence.

(a)

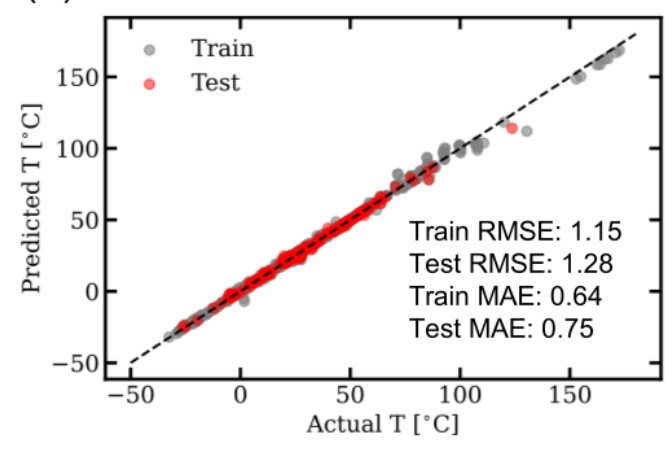

(b)

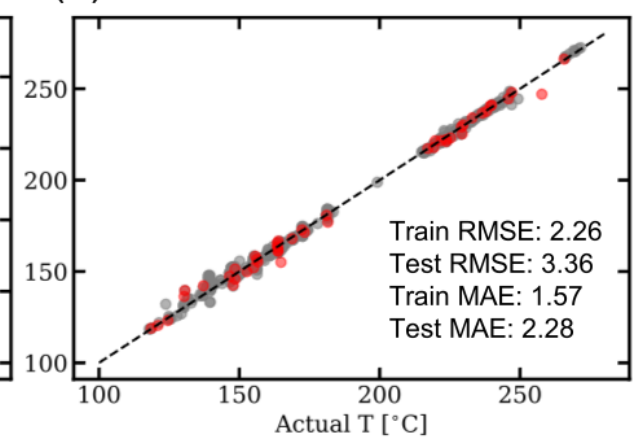

Figure S9. Parity plots for the DNN predictions on the training set (gray) and test set (red) for all (a) UCS and (b) LCS cloud point temperatures using 64-bit fingerprints as the solvent feature. RMSE and MAE values are reported in each plot. DNN architecture consists of three hidden layers with 256, 128, and 56 neurons per layer in sequence. 
(a)

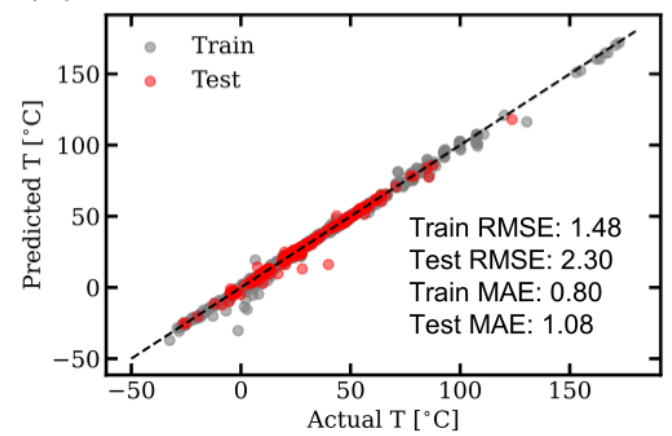

(b)

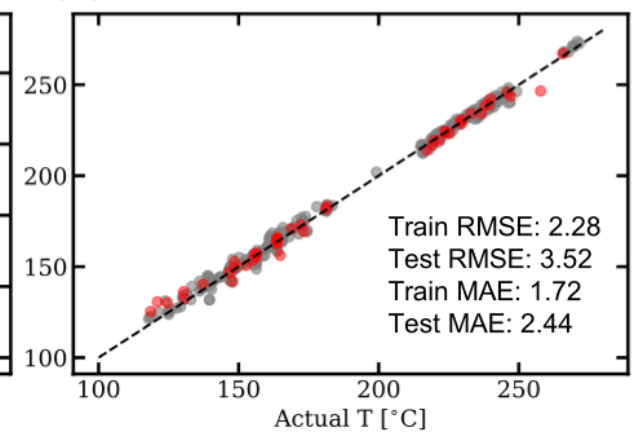

Figure S10. Parity plots for the DNN predictions on the training set (gray) and test set (red) for all (a) UCS and (b) LCS cloud point temperatures using the Hildebrand parameter as the solvent feature. RMSE and MAE values are reported in each plot. DNN architecture consists of three hidden layers with 256, 128, and 56 neurons per layer in sequence.

We also test the viability of using the Hildebrand solubility parameter as the solvent feature using the 3 hidden layer model. Figure S10 shows the parity plots for UCS and LCS predictions on the train and test data sets. We find that for the DNN model, the Hildebrand solvent feature performs slightly worse than using HSPs or fingerprint features. The test UCS predictions are increased by $1-2{ }^{\circ} \mathrm{C}$ compared to using HSPs, with the test RMSE within $4{ }^{\circ} \mathrm{C}$ for the LCS predictions. This is consistent with the five-fold cross-validation results (see Figures S11-S13 below). Comparing the Hildebrand trained model (Figure S13) with the HSP and fingerprint trained models (Figure S11 and S12, respectively) the test MAE values are consistently higher across all folds. While this increase may seem insignificant, the HSP solvent feature does not require additional computational time for the increased accuracy and thus, it is more favorable to use the HSP solvent feature if available. Additionally, more detailed features may be required when other polymers are introduced to the data set due to varying monomer-solvent interactions. Choosing the right set of features is crucial for optimal model performance.

Reducing the solvent feature from 64 inputs (fingerprint) to 3 inputs (HSPs) will reduce the time to train the model as discussed above. When building a ML pipeline, training time will affect the decision-making process during feature selection, model choice, and hyperparameter tuning. Although the time to train using fingerprints is only roughly $10 \%$ slower than using HSPs as solvent input, this will scale significantly with training set size. Nevertheless, the fingerprint 
feature can be generalized to other linear polymers or solvents, whereas HSPs are only available for a small number of polymer chemistries.

Five-fold cross-validation results are shown in Figures S11-S13 below and show how the DNN performs using various subsets of the PS data set to train the model. The data set is split into 5 folds, using 4 as the training data set and the remaining 1 as the test set. An additional 90:10 split of the combined 4 folds is used as the train and validation set. The DNN model is then trained 5 times with each run using a different test fold each time. As seen in Figure S11, the test RMSE values range between $1-2{ }^{\circ} \mathrm{C}$ and $3-5^{\circ} \mathrm{C}$ for the UCS and LCS data, respectively. This is slightly higher than that reported in Figure 1 of the manuscript, but the size of the test set is much larger and hence, the DNN model is trained on less data. Additionally, two of the folds in Figure S11 contain a single test point that is significantly increasing the RMSE value. Interestingly, the predicted test MAEs are consistently within 1 and $3{ }^{\circ} \mathrm{C}$ for the UCS and LCS predictions, respectively, for all folds. This implies that our model accuracy is robust regardless of the data in the test fold. Figure S12 shows that the DNN model trained with the 64-bit fingerprint solvent feature performs similarly well. However, the Hildebrand solvent feature (Figure S13) results show increased train and test RMSE values. 
UCS branch
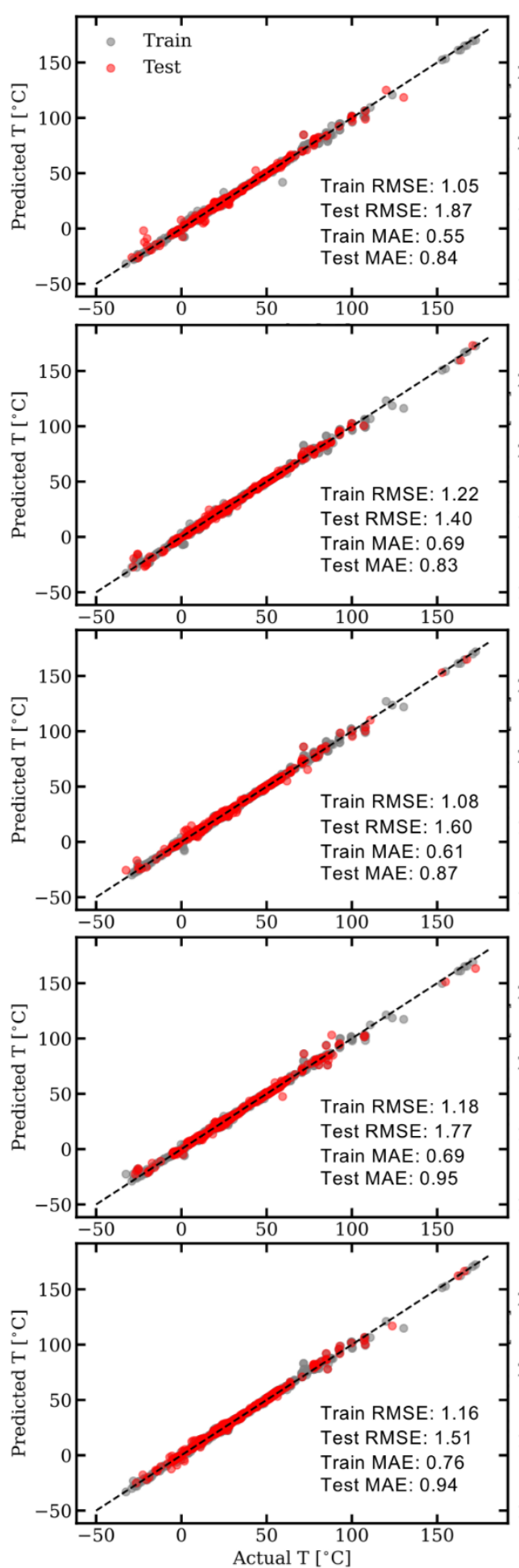

LCS branch
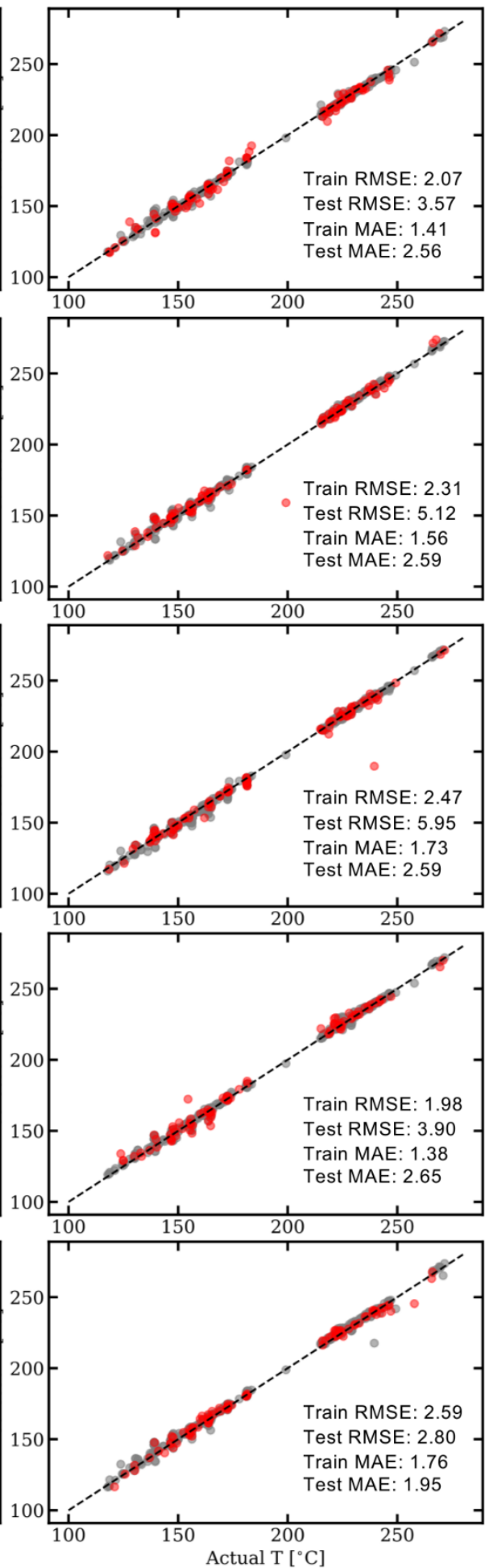

Figure S11. Top to bottom. Parity plots showing DNN predicted temperatures plotted against the observed (actual) temperatures using a different fold as the test set during training. Performance on the UCS (left) and LCS (right) data. HSPs are used as the solvent feature. 
UCS branch
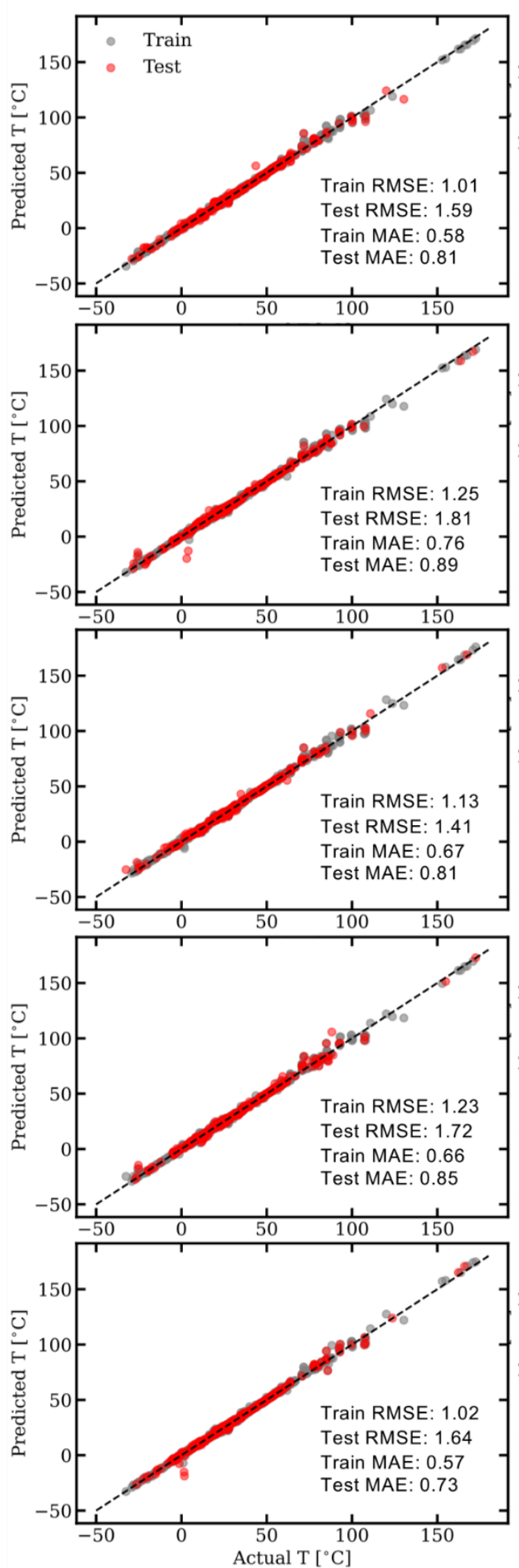

LCS branch
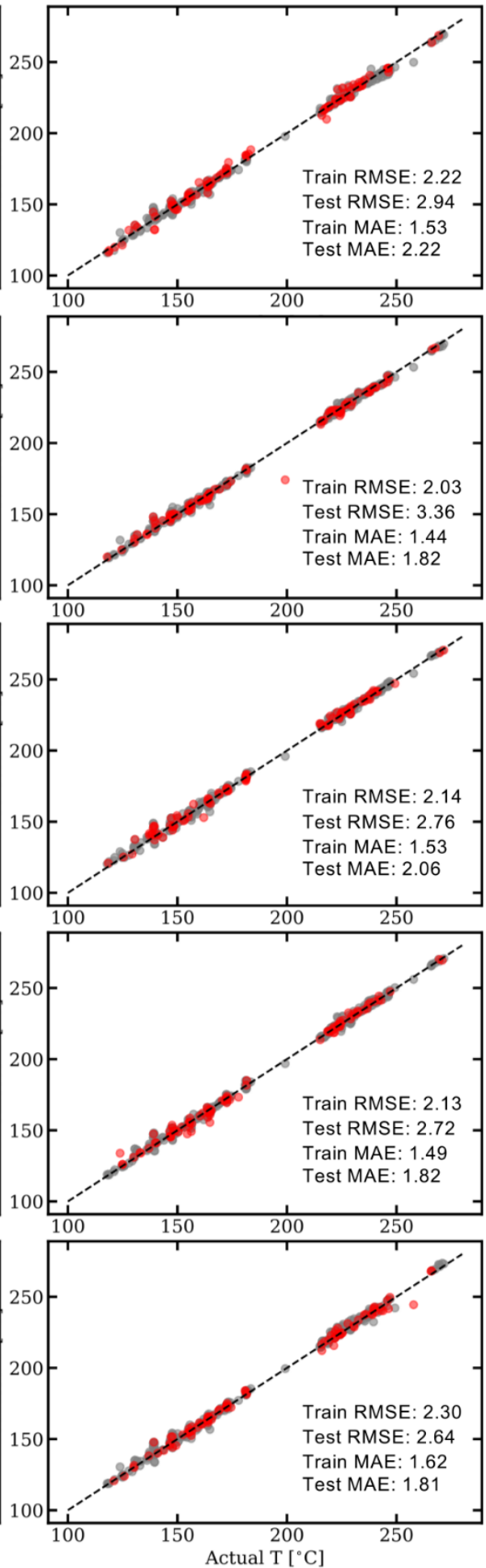

Figure S12. Top to bottom. Parity plots showing DNN predicted temperatures plotted against the observed (actual) temperatures using a different fold as the test set during training. Performance on the UCS (left) and LCS (right) data. Fingerprints (size $N=64$ bits) are used as the solvent feature. 
UCS branch
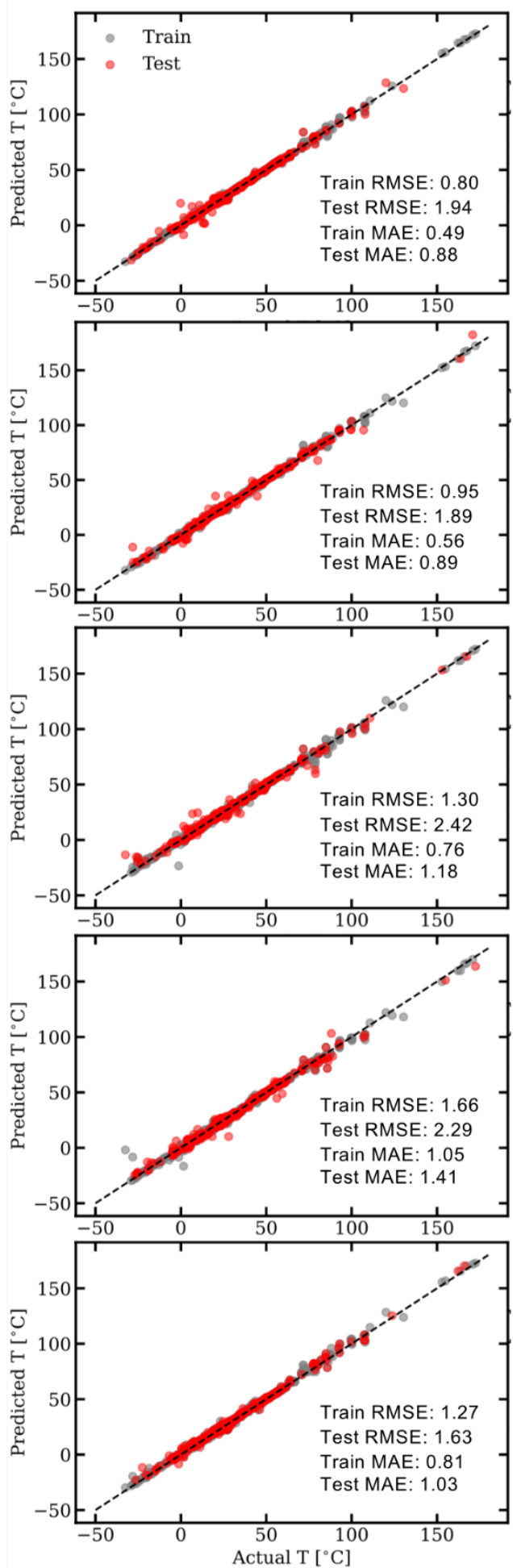

LCS branch
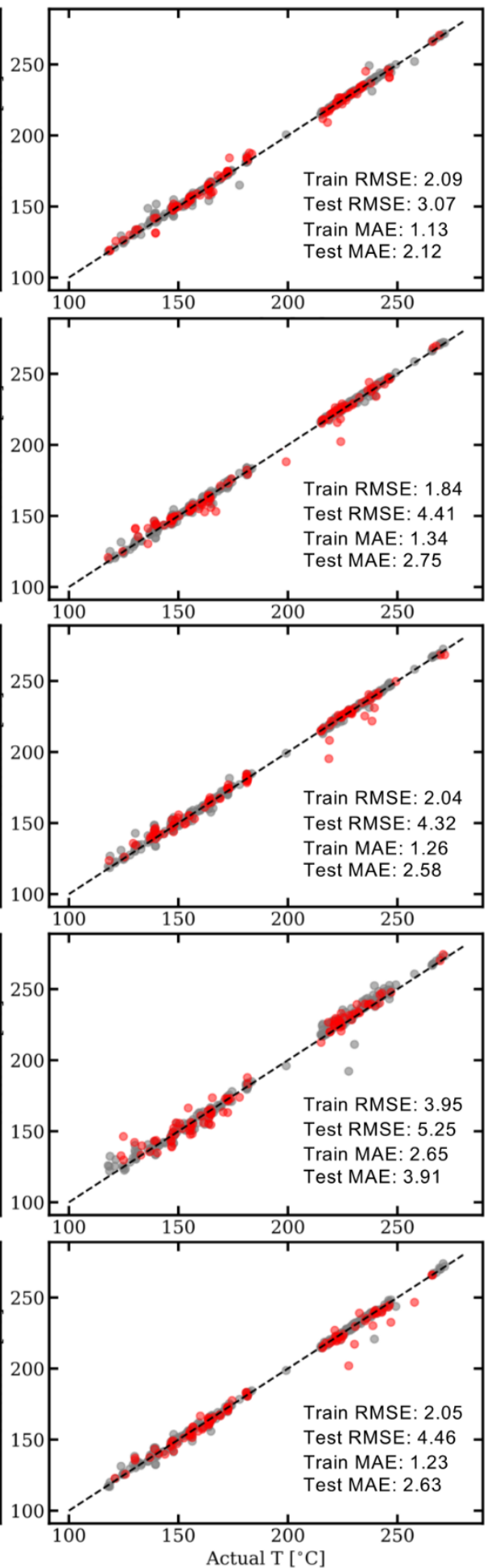

Figure S13. Top to bottom. Parity plots showing DNN predicted temperatures plotted against the observed (actual) temperatures using a different fold as the test set during training. Performance on the UCS (left) and LCS (right) data. Hildebrand parameters are used as the solvent feature. 


\section{Gaussian Process Regression}

\subsection{Model Details}

Aside from the neural network model, we also train a Gaussian process regression (GPR) model on the polystyrene data set. This is a nonparametric model that determines all possible functions that fit the training data. The model specifies a GP prior distribution on the latent function,

$$
f(\boldsymbol{x}) \sim G P\left(m(\boldsymbol{x}), k\left(\boldsymbol{x}, \boldsymbol{x}^{\prime}\right)\right)
$$

where $m(\boldsymbol{x})$ is the mean function (assumed to be constant and zero) and $k\left(\boldsymbol{x}, \boldsymbol{x}^{\prime}\right)$ is the kernel (covariance) function that controls the smoothness of the function. The training data is then used to maximize the log-marginal-likelihood to determine the posterior distribution. In our work, we use the constant kernel combined with the radial basis function (RBF),

$$
k\left(\boldsymbol{x}, \boldsymbol{x}^{\prime}\right)=\sigma_{f}^{2} \exp \left(-\frac{\left\|x-\boldsymbol{x}^{\prime}\right\|^{2}}{2 l^{2}}\right)
$$

where $\sigma_{f}^{2}$ is the signal variance and $l$ is the length scale, both to be optimized. The RBF kernel is a common kernel used to control the smoothness of the functions, meaning similar input values will result in similar outputs and smooth curves. We can also specify a noise level in the target values by varying the parameter $\alpha$, which is added to the diagonal of the kernel matrix during training (i.e., larger values increase the noise level of the observations). In other words, the $\alpha$ parameter is the variance of the Gaussian noise on the training observations. During training we use the 'L-BFGS-B' optimizer to tune the above hyperparameters, restarting the optimizer 10 times with a different initialization each time.

To simulate intelligent exploration of phase space (see Figure 3 of the manuscript), 5 random samples are drawn from the PS-cyclohexane data set and cloud points are revealed to train the GPR model. The remaining 977 cloud points are used to test the performance of the model. After training the model on the first 5 cloud points and calculating $R^{2}$ and RMSE on the test set, a sample is removed from the test set and added to the training data set to re-train the model. This procedure mimics a true experiment in which we wish to run cloud point measurements until our model can predict the remaining unknown samples within experimental uncertainty. The sample that is drawn from the test data set is either i) random or ii) the prediction with the largest 
uncertainty from the GPR model (exploration). We run this ML experiment 20 times with different initial random samples and average the results over all trials. Predictions of the GPR model trained after 50 samples can be found in Section 6.3 below.

\subsection{GPR Model Pe rformance}

Figure S14 explores the performance of the GPR model with varying $\alpha$ values. For this model, we use a 90:10 stratified split to train and test the model using the HSP solvent features. As we increase $\alpha$ from 0.5 to 3, we find that the difference between the test and train RMSE decreases, indicating less overfitting with increasing $\alpha$. Additionally, $\alpha$ values greater than 3 did not improve performance significantly and thus, we choose $\alpha=3.0$ for training on the entire data set. Note that we set a smaller $\alpha=0.5$ to simulate the PS/cyclohexane phase space exploration (Figure 3 of the manuscript) to reduce significant bias in the sample selection process.

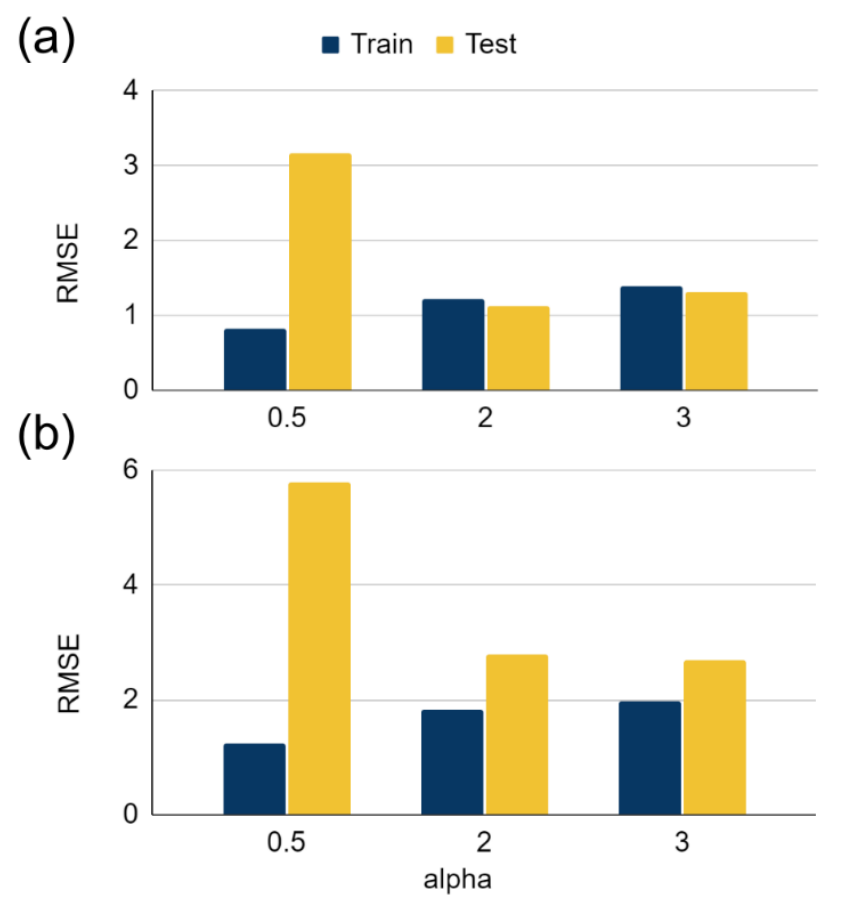

Figure S14. RMSE measured from temperature predictions on the training set (blue) and test set (yellow) for (a) UCS and (b) LCS data and various $\alpha$ values. 
(a)

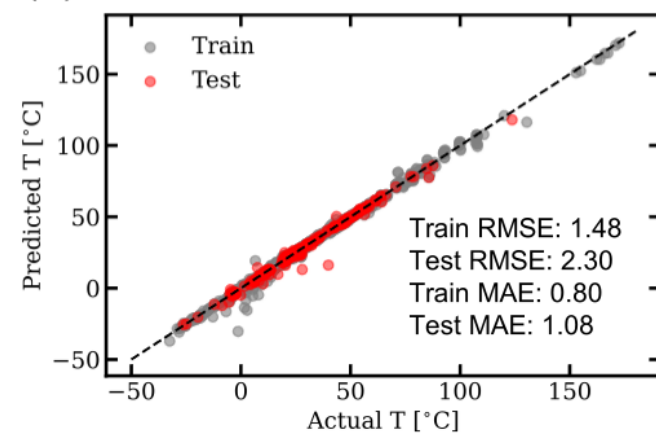

(b)

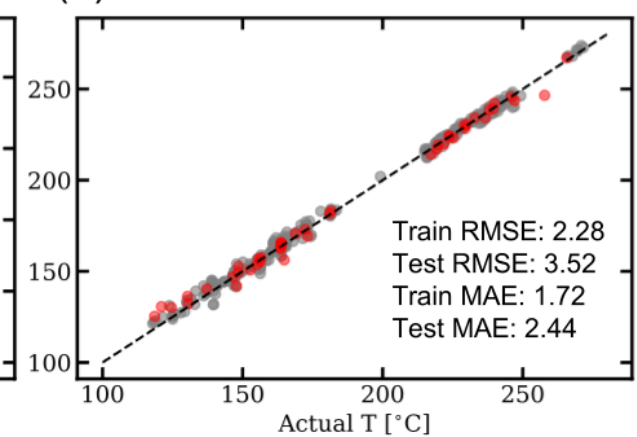

Figure S15. Parity plots for the GPR predictions using the HSP solvent feature on the training set (gray) and test set (red) for all (a) UCS and (b) LCS cloud point temperatures. RMSEand MAE values are reported in each plot. GPR model trained using the constant kernel combined with the radial basis function (RBF) kernel as the covariance function.

The performance of the GPR model is summarized in Figure S15. As shown in the parity plots in Figure S15a and b, the model predicts the test data with a MAE less than $1{ }^{\circ} \mathrm{C}$ for the UCS plot and $2{ }^{\circ} \mathrm{C}$ for the LCS data, similar to the DNN model. One significance to using the RBF kernel is that we can predict smoother curves, as values close in input space will have similar output predictions. This can be seen by the predicted coexistence curves for select solvents, including isopleths and predictions for unknown cyclopentane $M_{\mathrm{w}}$, in Figure S16 below. A significant tradeoff with the GPR model is that training time scales with increasing training set size, making it unfavorable for large data sets. With our polystyrene data set the GPR model is an order of magnitude slower to train than the DNN model (depends on the number of epochs for the DNN model). However, there is a wealth of information that the GPR model can provide that the DNN model cannot. For instance, the GPR model provides uncertainty in the predicted cloud points, which may be used to improve performance by targeting specific polymer solutions or for use in high-throughput experimentation.

Similar to Figure 2 in the manuscript, we plot the predicted cloud point temperatures against the observed temperatures for select solvents in Figure S16. These predicted curves appear to be significantly smoother than the predictions from the DNN. For instance, the predictions for cyclohexane (UCS, Figure S16a) and tert-butyl acetate (LCS, Figure S16b) show excellent agreement with the observed data and exhibit the typical UCS/LCS curve shapes found in experiments and polymer solution theory. Furthermore, we find good agreement in the predictions 
of methyl acetate (Figure S16c and d) and LCS temperatures for proprionitrile isopleths (Figure S16e). One advantage the GPR model has over the DNN model is the ability to provide uncertainty measurements in the predicted temperatures. In predicting new unknown cloud points for cyclopentane in Figure S16f, we find that the newly measured cloud point temperatures (that did not originate from the polystyrene data set) are well within the predicted uncertainty range.

Five-fold cross-validation is used to determine the average performance using various subsets of the polystyrene data set to train the GPR model. We split the polystyrene data set into five folds, using 4 of the folds to train the model and the remaining 1 to test the performance on unseen data. This is repeated 5 times using a different fold for the test set each time. Figure S17 shows the cross-validation results for each test fold. The results show that the test RMSE values are consistently around $2{ }^{\circ} \mathrm{C}$ and $3.5^{\circ} \mathrm{C}$ on average for the UCS and LCS predictions, respectively. There is more variability in the LCS data due to the overwhelmingly smaller training size compared to the UCS cloud points (see Figure S1). Nevertheless, the test MAEs are all within 1 and $2{ }^{\circ} \mathrm{C}$, respectively, and are slightly smaller than the DNN model using the same feature vector.

Mean absolute error for each solvent in the test data set is shown in Figure S18. Similar to the performance of the DNN model, we find that the solvents with less data have a slightly larger MAE. We find that most of the solvents have an average error of less than $3{ }^{\circ} \mathrm{C}$, which is in agreement with the DNN model (see Figure S7). Additionally, we show in Figure S16 and Figures S19-S22 that the GPR model can predict a much smoother solubility curve shape compared to the DNN predictions. Thus, combined with the results in Figure 3 of the manuscript, the GPR model is an excellent model choice for active learning of polymer solution phase behavior. 
(a)

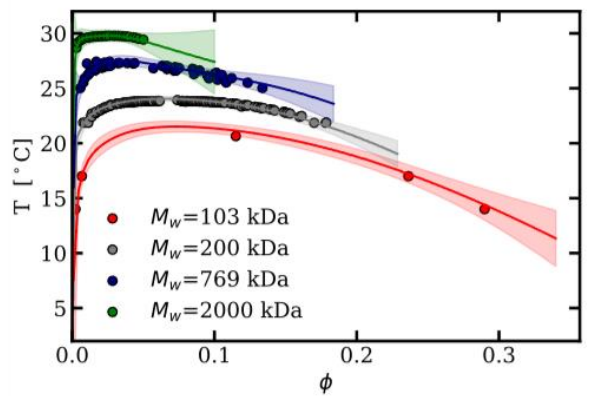

(c)

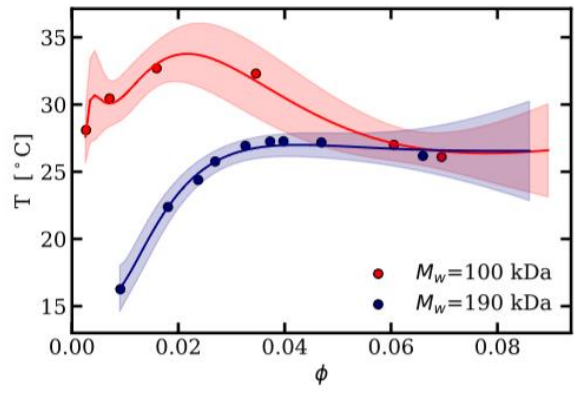

(e)

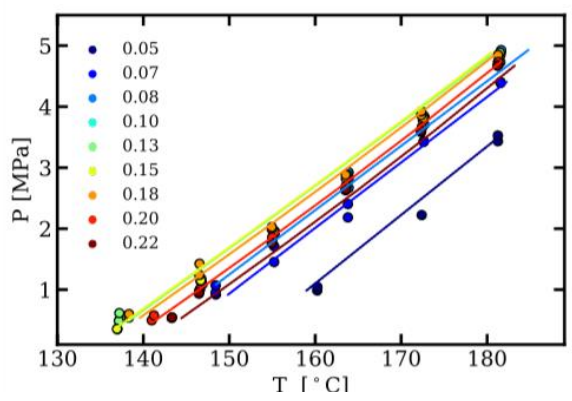

(b)

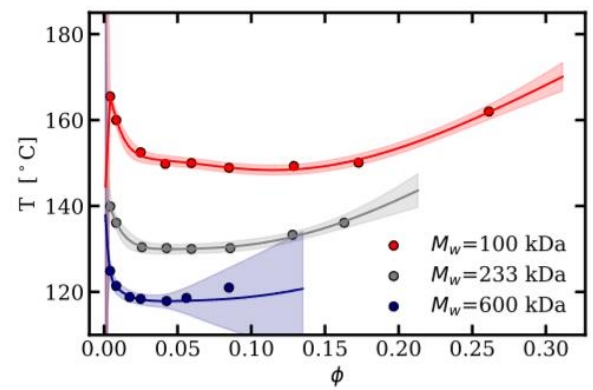

(d)

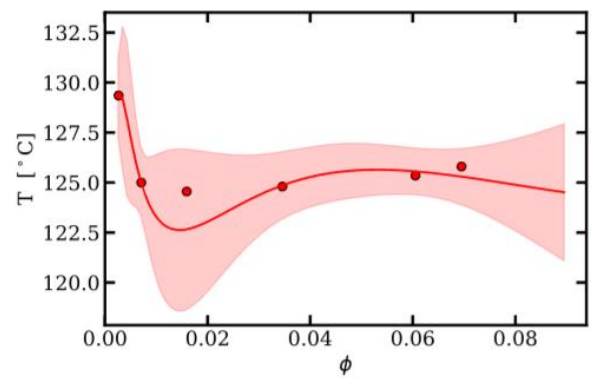

(f)

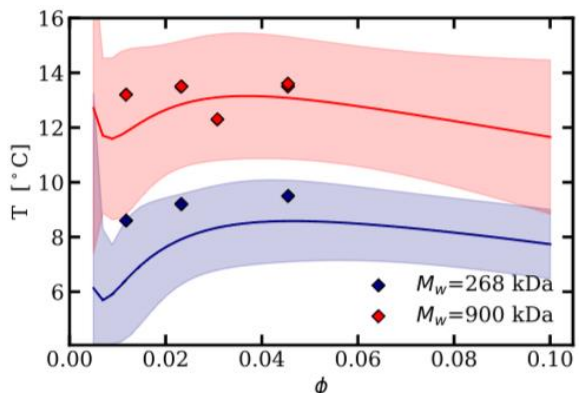

Figure S16. GPR predictions (lines) for select solvents compared to observed data (markers). Cloud point temperatures as a function of PS volume fraction in methyl ac etate for (a) UCS temperatures and (b) LCS temperatures. (c) LCS pressure plotted against the predicted cloud point temperature at constant polymer volume fraction (isopleths). (d) Cyclopentane cloud points determined by UV-vis spectroscopy compared to the GPR model prediction for two unknown molecular weights. Shaded regions show one standard deviation from the mean. 
UCS branch
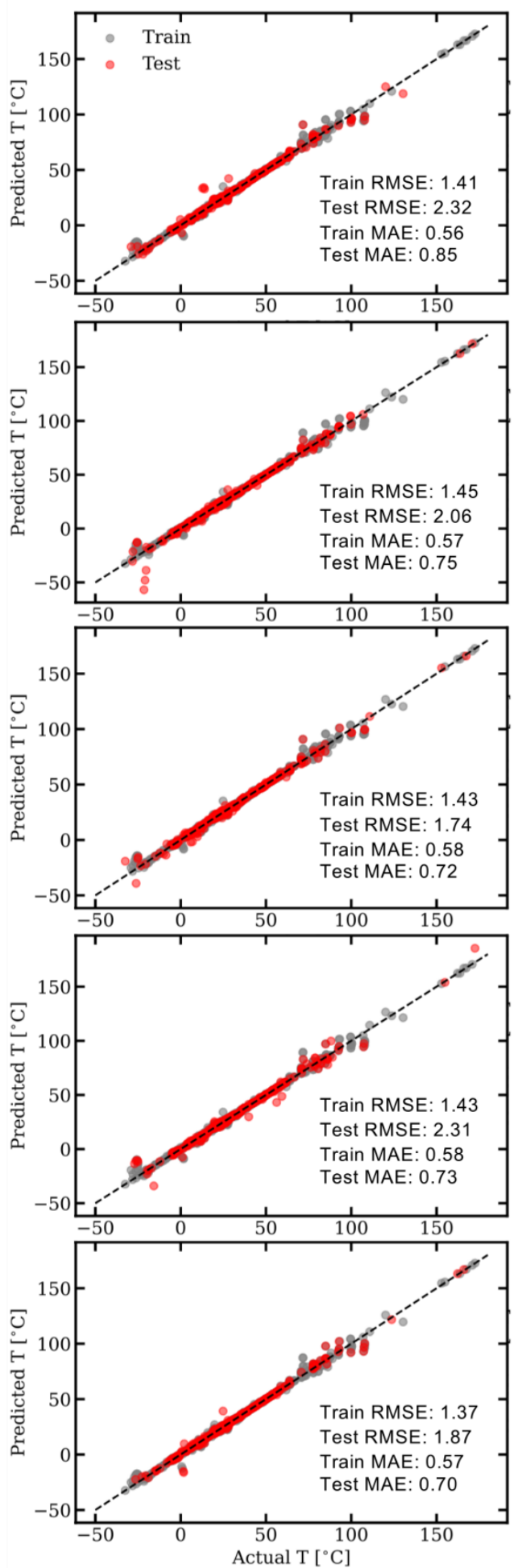

LCS branch
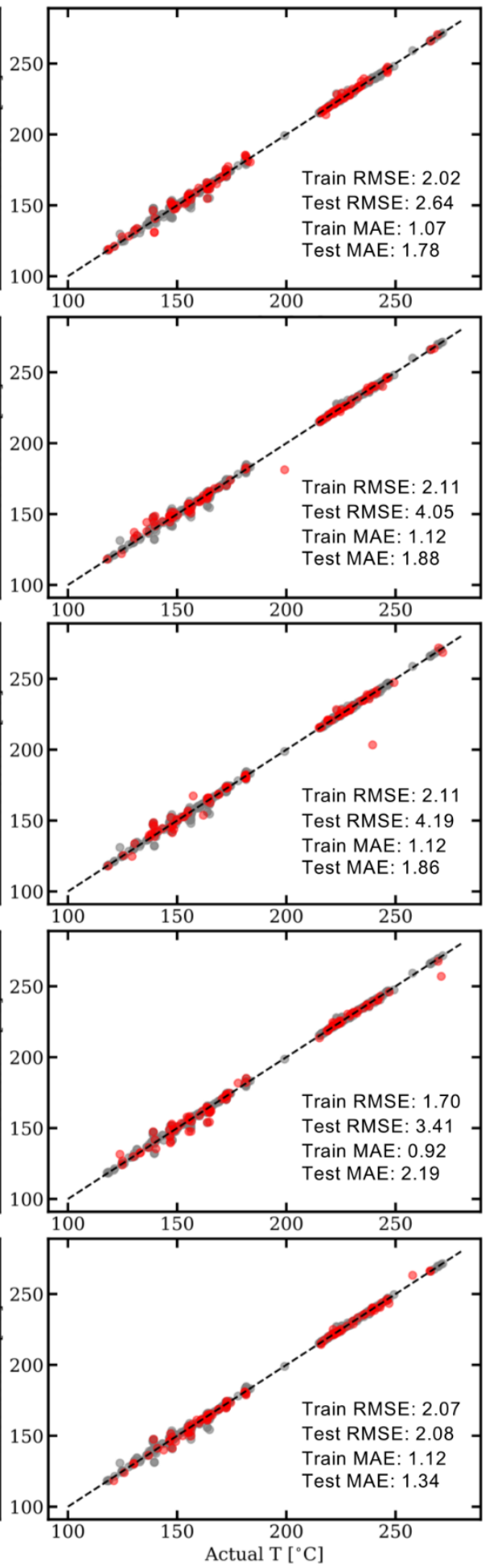

Figure S17. Top to bottom. Parity plots showing GPR predicted temperatures plotted against the observed (actual) temperatures using a different fold as the test set during training. Performance on the UCS (left) and LCS (right) data. HSPs are used as the solvent feature. 


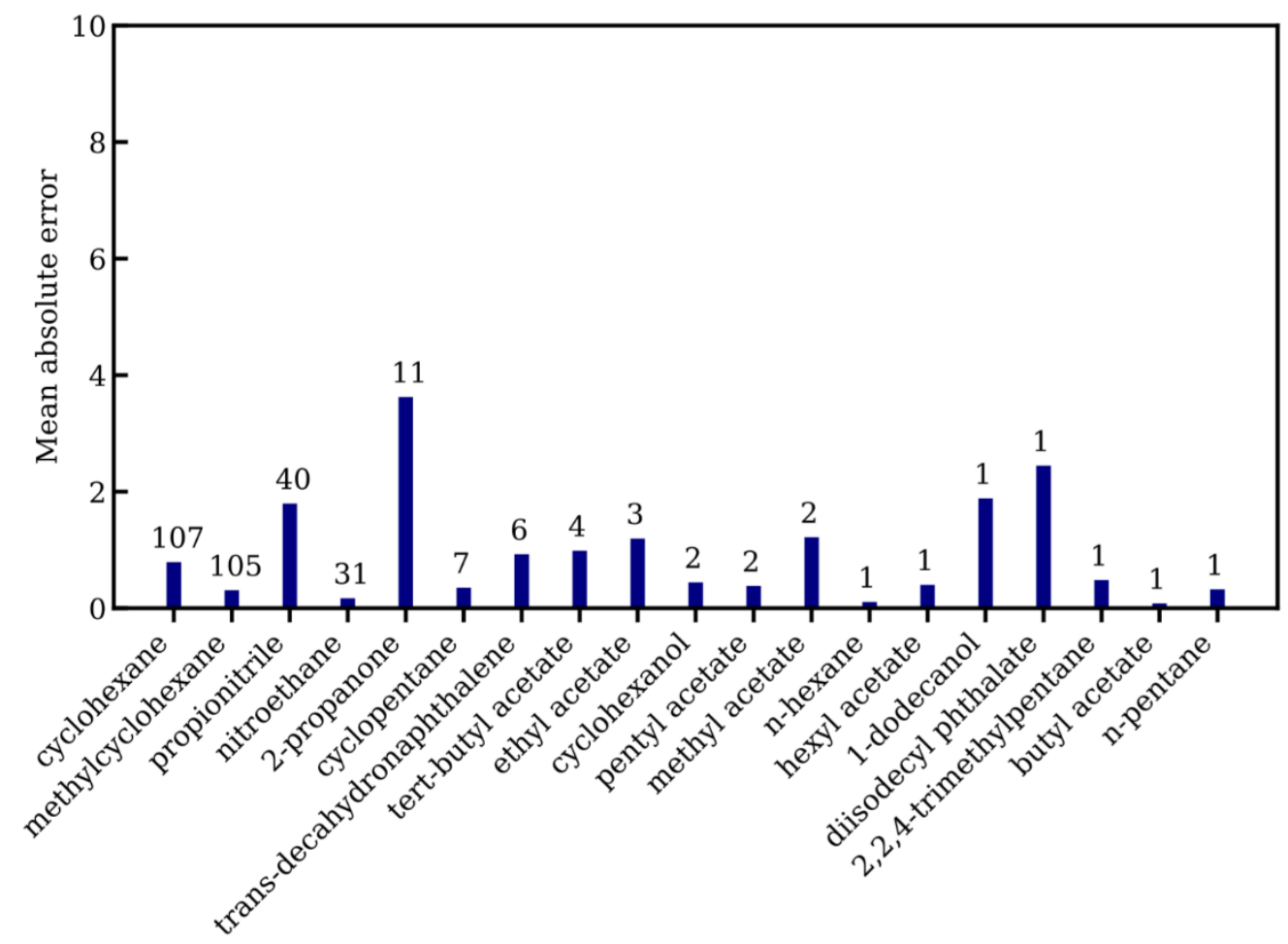

Figure S18. Mean absolute error by solvent on the test data set for the GPR model using HSP values for the solvent feature.

\subsection{Predictions for PS/Cyclohexane}

In Figure 3 of the main manuscript, we show RMSE, $R^{2}$, and number of sampled molecular weights during phase space exploration using the GPR model $(\alpha=0.5)$. After 50 cloud points are added to the training data set, predictions for various $M_{\mathrm{w}}$ values of PS in cyclohexane are calculated and plotted in Figures S19-S22 below. We choose the model with the closest $R^{2}$ to the average $(0.77$ and 0.88 for the random and "exploration" algorithms, respectively). In most cases, we find that the predictions using intelligent exploration are in much better agreement with the experimental data than randomly sampling the phase space. Additionally, the curve shapes are akin to the UCStype curves found experimentally. Thus, these results show that active learning with the GPR model is possible to elucidate polymer/solvent phase behavior with a minimal number of required experiments. 
(a)

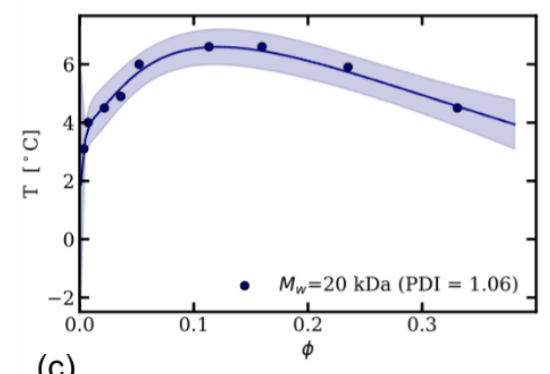

(c)

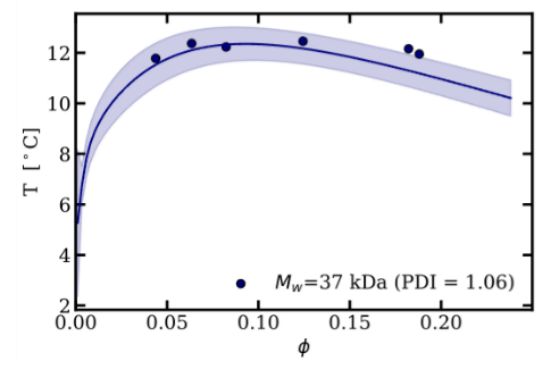

(b)

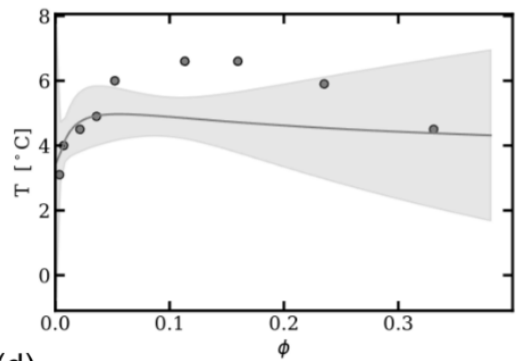

(d)

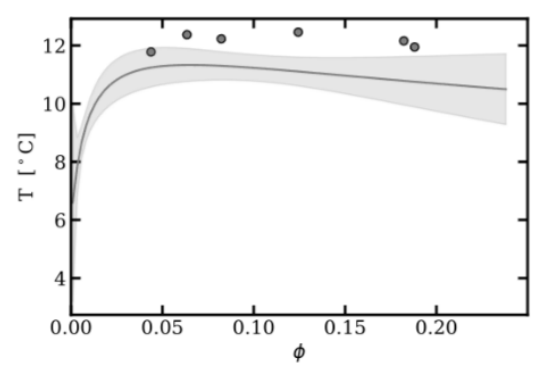

Figure S19. Polystyrene phase behavior in cyclohexane (top row, $M_{\mathrm{w}}=20 \mathrm{kDa}$ and bottom, $M_{\mathrm{w}}=37 \mathrm{kDa}$ ) at atmospheric pressure predicted using the GPR model (line) compared to experimental data (markers). (a) and (c) show the GPR model predictions trained on 50 samples using GPR-guided sampling (exploration), whereas (b) and (d) show predictions trained from 50 random samples. Shaded regions show one standard deviation from the mean.

(a)

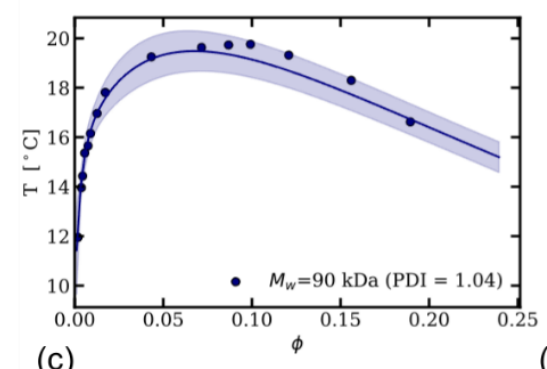

(c)

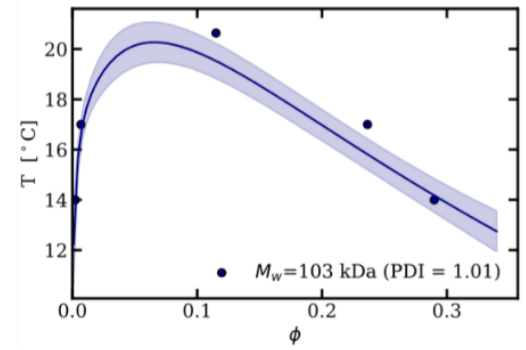

(b)

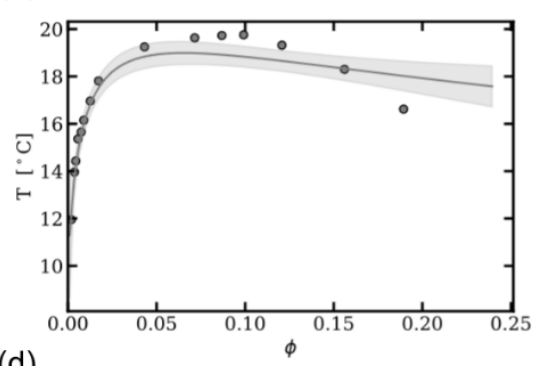

(d)

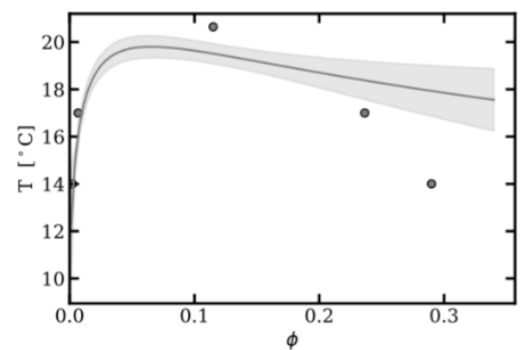

Figure S20. Polystyrene phase behavior in cyclohexane (top row, $M_{\mathrm{w}}=90 \mathrm{kDa}$ and bottom, $M_{\mathrm{w}}=103 \mathrm{kDa}$ ) at atmospheric pressure predicted using the GPR model (line) compared to experimental data (markers). (a) and (c) show the GPR model predictions trained on 50 samples using GPR-guided sampling (exploration), whereas (b) and (d) show predictions trained from 50 random samples. Shaded regions show one standard deviation from the mean. 
(a)

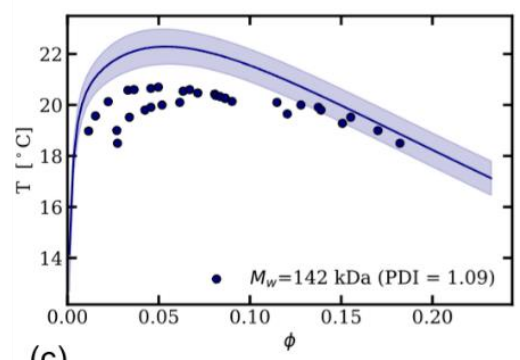

(c)

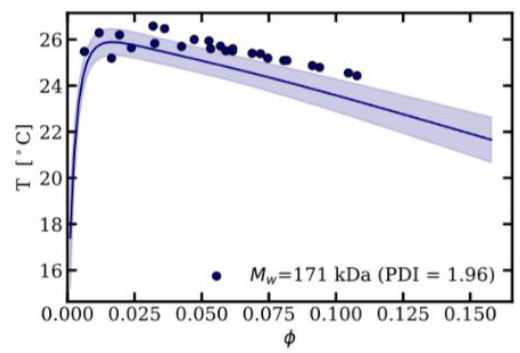

(b)

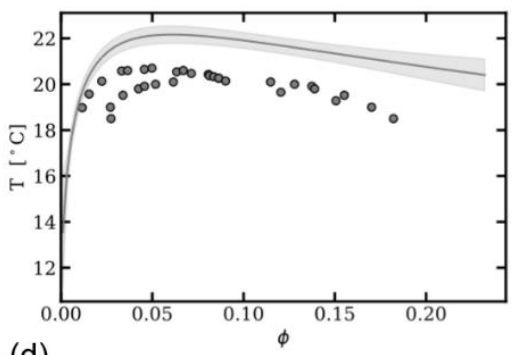

(d)

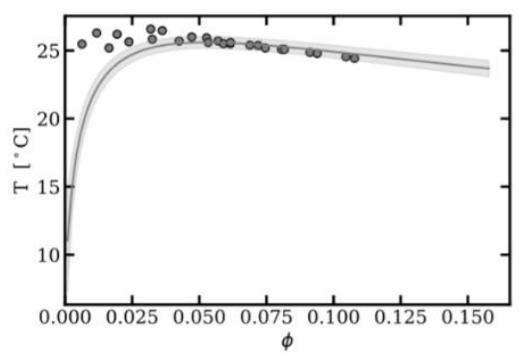

Figure S21. Polystyrene phase behavior in cyclohexane (top row, $M_{\mathrm{w}}=142 \mathrm{kDa}$ and bottom, $M_{\mathrm{w}}=171$ $\mathrm{kDa}$ ) at atmospheric pressure predicted using the GPR model (line) compared to experimental data (markers). (a) and (c) show the GPR model predictions trained on 50 samples using GPR-guided sampling (exploration), whereas (b) and (d) show predictions trained from 50 random samples. Shaded regions show one standard deviation from the mean.

(a)

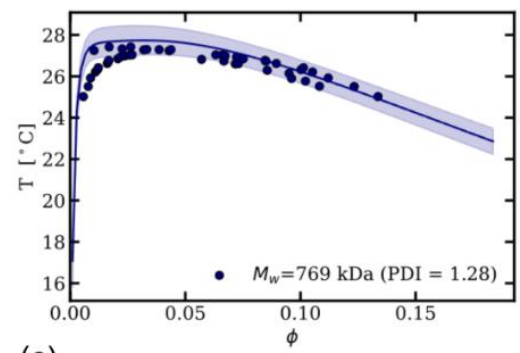

(c)

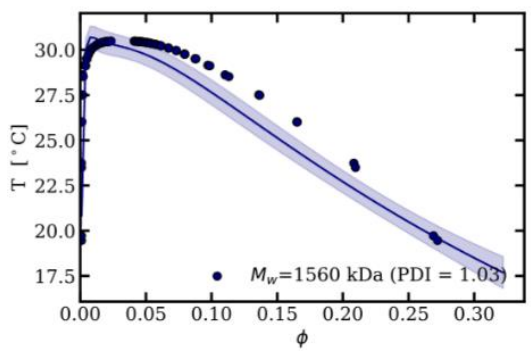

(b)

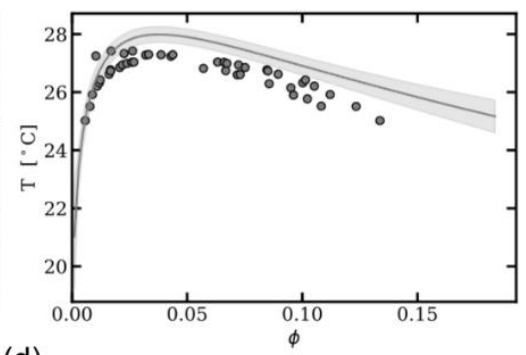

(d)

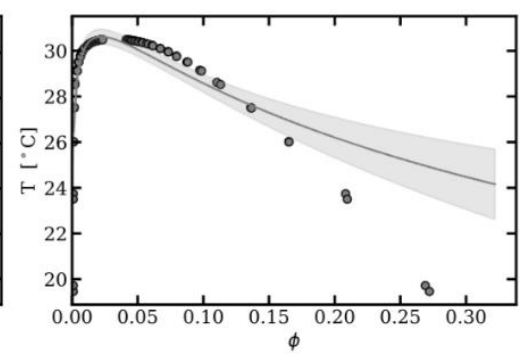

Figure S22. Polystyrene phase behavior in cyclohexane (top row, $M_{\mathrm{w}}=769 \mathrm{kDa}$ and bottom, $M_{\mathrm{w}}=1560$ $\mathrm{kDa}$ ) at atmospheric pressure predicted using the GPR model (line) compared to experimental data (markers). (a) and (c) show the GPR model predictions trained on 50 samples using GPR-guided sampling (exploration), whereas (b) and (d) show predictions trained from 50 random samples. Shaded regions show one standard deviation from the mean. 


\section{Experimental Details: PS in Cyclopentane}

Polystyrene cloud points in cyclopentane were determined using a Cary 5000 UV-vis spectrophotometer with a Peltier temperature controller. Two polystyrene samples were examined: one with $M_{\mathrm{w}}=268 \mathrm{kDa}(\mathrm{PDI}=1.07)$ and a standard with $M_{\mathrm{w}}=900 \mathrm{kDa}(\mathrm{PDI}=1.06)$. Both polymers were obtained from Polymer Source and cyclopentane was obtained from Sigma Aldrich. Solutions containing 1 to 5 volume percent polystyrene in cyclopentane were prepared for each molecular weight and vortexed for 5 minutes to ensure that the PS was properly dissolved. Solutions were then placed in $1 \mathrm{~cm}$ thickness quartz cuvettes for UV-vis measurements and cooled from room temperature to below their cloud points. During these temperature ramps, a gentle stream of dry nitrogen was blown over the samples to prevent water from condensing on the cuvettes and creating an increase in absorption that could be mistaken for the polymer cloud point. A relatively slow cooling rate of $0.5{ }^{\circ} \mathrm{C} / \mathrm{min}$ was used to minimize hysteresis and an absorption of light with wavelength $700 \mathrm{~nm}$ was used to detect the cloud transition (a sharp increase in absorption intensity occurred at this temperature, and the solutions could be observed afterwards to have become milky and opaque). The temperature at which absorption intensity began to increase was taken as the cloud point. The entire transition took place over a temperature range of $1{ }^{\circ} \mathrm{C}$ or less. 


\section{Additional Computational Details}

All models were trained on an 8th Generation Intel Core i7-8850H CPU with 64GB of RAM. All results were obtained using Python 3.7 with Tensorflow and Scikit-Learn. Note that the reported RMSE and MAE values will change slightly with a change in CPU architecture and software/package versions due to floating point arithmetic. 


\section{References}

(1) Wohlfarth, C. CRC Handbook of Liquid-Liquid Equilibrium Data of Polymer Solutions; CRC Press, 2007.

(2) Kim, S.; Chen, J.; Cheng, T.; Gindulyte, A.; He, J.; He, S.; Li, Q.; Shoemaker, B. A.; Thiessen, P. A.; Yu, B.; others. PubChem 2019 Update: Improved Access to Chemical Data. Nucleic Acids Res. 2019, 47 (D1), D1102--D1109.

(3) Hildebrand, J. H.; Scott, R. L. The Solubility of Nonelectrolytes; Reinhold Publishing Corporation, 1950.

(4) Hildebrand, J. H.; Scott, R. L. Regular Solutions; Prentice-Hall, 1962.

(5) Hansen, C. M. The Three Dimensional Solubility Parameter. Danish Tech. Copenhagen 1967,14 .

(6) Venkatram, S.; Kim, C.; Chandrasekaran, A.; Ramprasad, R. Critical Assessment of the Hildebrand and Hansen Solubility Parameters for Polymers. J. Chem. Inf. Model. 2019, 59 (10), 4188-4194.

(7) Barnett, J. W.; Bilchak, C. R.; Wang, Y.; Benicewicz, B. C.; Murdock, L. A.; Bereau, T.; Kumar, S. K. Designing Exceptional Gas-Separation Polymer Membranes Using Machine Learning. Sci. Adv. 2020, 6 (20), eaaz4301.

(8) Chandrasekaran, A.; Kim, C.; Venkatram, S.; Ramprasad, R. A Deep Learning SolventSelection Paradigm Powered by a Massive Solvent/Nonsolvent Database for Polymers. Macromolecules 2020.

(9) RDKit: Open-Source Cheminformatics Software.

(10) LeCun, Y. A.; Bottou, L.; Orr, G. B.; Müller, K.-R. Efficient Backprop. In Neural networks: Tricks of the trade; Springer, 2012; pp 9-48. 